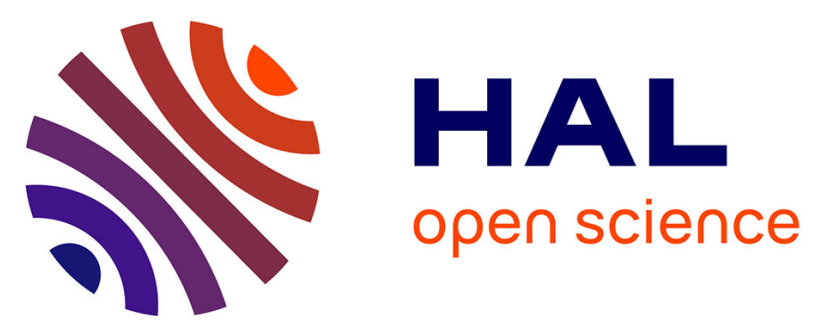

\title{
Mechanism of Edge Localized Mode Mitigation by Resonant Magnetic Perturbations
}

Marina Becoulet, F. Orain, G. T. A. Huijsmans, S. Pamela, P. Cahyna, M. Hoelzl, X. Garbet, E. Franck, E. Sonnendrücker, Guilhem Dif-Pradalier, et al.

\section{- To cite this version:}

Marina Becoulet, F. Orain, G. T. A. Huijsmans, S. Pamela, P. Cahyna, et al.. Mechanism of Edge Localized Mode Mitigation by Resonant Magnetic Perturbations. Physical Review Letters, 2014, 113 (11), 10.1103/PhysRevLett.113.115001 . hal-02184565

\section{HAL Id: hal-02184565 https://hal.science/hal-02184565}

Submitted on 16 Jul 2019

HAL is a multi-disciplinary open access archive for the deposit and dissemination of scientific research documents, whether they are published or not. The documents may come from teaching and research institutions in France or abroad, or from public or private research centers.
L'archive ouverte pluridisciplinaire HAL, est destinée au dépôt et à la diffusion de documents scientifiques de niveau recherche, publiés ou non, émanant des établissements d'enseignement et de recherche français ou étrangers, des laboratoires publics ou privés. 


\title{
Mechanism of Edge Localized Mode Mitigation by Resonant Magnetic Perturbations
}

\author{
M. Bécoulet, ${ }^{1}$ F. Orain, ${ }^{1}$ G. T. A. Huijsmans, ${ }^{2}$ S. Pamela, ${ }^{3}$ P. Cahyna, ${ }^{4}$ M. Hoelzl, ${ }^{5}$ X. Garbet, ${ }^{1}$ \\ E. Franck, ${ }^{5}$ E. Sonnendrücker, ${ }^{5}$ G. Dif-Pradalier, ${ }^{1}$ C. Passeron, ${ }^{1}$ G. Latu, ${ }^{1}$ J. Morales, ${ }^{1}$ E. Nardon, ${ }^{1}$ \\ A. Fil,${ }^{1}$ B. Nkonga, ${ }^{6}$ A. Ratnani, ${ }^{6}$ and V. Grandgirard ${ }^{1}$ \\ ${ }^{1}$ CEA, IRFM, 13108 Saint-Paul-Lez-Durance, France \\ ${ }^{2}$ ITER Organization, Route de Vinon-sur-Verdon, 13067 Saint-Paul-Lez-Durance, France \\ ${ }^{3}$ CCFE, Culham Science Centre, Oxon OX14 3DB, United Kingdom \\ ${ }^{4}$ Institute of Plasma Physics ASCR, 18200 Prague 8, Czech Republic \\ ${ }^{5}$ Max-Planck-Institut, 85748 Garching, Germany \\ ${ }^{6}$ Laboratoire de Mathématiques J.A. Dieudonné, UMR 7351, CNRS UNS, Université de Nice-Sophia Antipolis, \\ Parc Valrose, 06108 Nice Cedex 02, France \\ (Received 11 March 2014; published 8 September 2014)
}

\begin{abstract}
A possible mechanism of edge localized modes (ELMs) mitigation by resonant magnetic perturbations (RMPs) is proposed based on the results of nonlinear resistive magnetohydrodynamic modeling using the JOREK code, realistic JET-like plasma parameters and an RMP spectrum of JET error-field correction coils (EFCC) with a main toroidal number $n=2$ were used in the simulations. Without RMPs, a large ELM relaxation is obtained mainly due to the most unstable medium- $n$ ballooning mode. The externally imposed RMP drives nonlinearly the modes coupled to $n=2$ RMP which produce small multimode relaxations, mitigated ELMs. The modes driven by RMPs exhibit a tearinglike structure and produce additional islands. Mitigated ELMs deposit energy into the divertor mainly in the structures ("footprints") created by $n=2$ RMPs, however, slightly modulated by other nonlinearly driven even harmonics. The divertor power flux during a ELM phase mitigated by RMPs is reduced almost by a factor of 10. The mechanism of ELM mitigation by RMPs proposed here reproduces generic features of high collisionality RMP experiments, where large ELMs are replaced by small, much more frequent ELMs or magnetic turbulence. Total ELM suppression was also demonstrated in modeling at higher RMP amplitude.
\end{abstract}

DOI: 10.1103/PhysRevLett.113.115001

PACS numbers: 52.25.Xz, 52.25.Fi, 52.30.Cv

Introduction.-The aim of the ITER project is the demonstration of the scientific feasibility of a nuclear fusion reactor based on a magnetic confinement concept as a future source of energy [1]. Plasma edge magnetohydrodynamic (MHD) instabilities, such as edge localized modes (ELMs) driven by the pressure gradient and the plasma current, produce quasiperiodic relaxations of the edge density and temperature profiles on few hundred microseconds time scale. ELMs in ITER are predicted to lead to the transient heat fluxes reaching tens of $\mathrm{GW} \mathrm{m}^{-2}$ which would cause strong erosion of the plasma-facing component materials [1-2]; hence, ELM control is mandatory in ITER. Recently the application of resonant magnetic perturbations (RMPs) demonstrated the possibility of total ELMs suppression or strong mitigation of their size [3-9], motivating the use of this method in ITER [1]. In the last decade, the nonlinear MHD theory and modeling have made significant progress to refine the understanding of ELM [10-14] and RMP [15-20] physics. However, the understanding of RMP interaction with ELMs was still missing and had not yet been modeled, motivating the work we are presenting here. One can clearly distinguish two groups of RMP experiments. At high collisionality the application of RMPs usually leads to the replacement of large ELMs by small frequent ELMs or MHD turbulence, and the divertor power loads are reduced by RMPs. Small changes in edge pressure gradient and MHD stability are reported [3-8]. On the other hand, at low collisionality resulting from strong density pump-out due to RMPs, ELMs have been totally suppressed in DIII-D, and the plasma edge appears to be stable to peeling-ballooning modes [9].

In the present Letter we report on the first observation of ELM mitigation by RMPs in nonlinear resistive MHD simulations done with the code JOREK in toroidal geometry, including confined plasma region, $X$ point, scrape off layer (SOL), and divertor [14]. The results we found in modeling correspond to generic features observed in high collisionality RMP experiments. The JOREK model used here includes two-fluid diamagnetic effects important in the pedestal region and is described in detail in [18]. The realistic JET pulse 77329 parameters used as in $[17,18,19]$ are $R_{0}=2.9 \mathrm{~m}, a=0.89 \mathrm{~m}, B_{\text {tor }}=1.8 \mathrm{~T}, q_{95}=3.8$. The density and temperature profiles were approximated with the following central and pedestal values: $T_{\mathrm{e}}(0)=6 \mathrm{keV}$, $T_{e, \text { ped }}=1.8 \mathrm{keV}, T_{i}=T_{e}, n_{e}(0)=5 \times 10^{19} \mathrm{~m}^{-3}, n_{e, \text { ped }}=$ $3.3 \times 10^{19} \mathrm{~m}^{-3}$. The toroidal rotation profile was taken parabolic with a central frequency $\Omega_{\phi}(0)=38 \mathrm{krad} / \mathrm{s}$. 


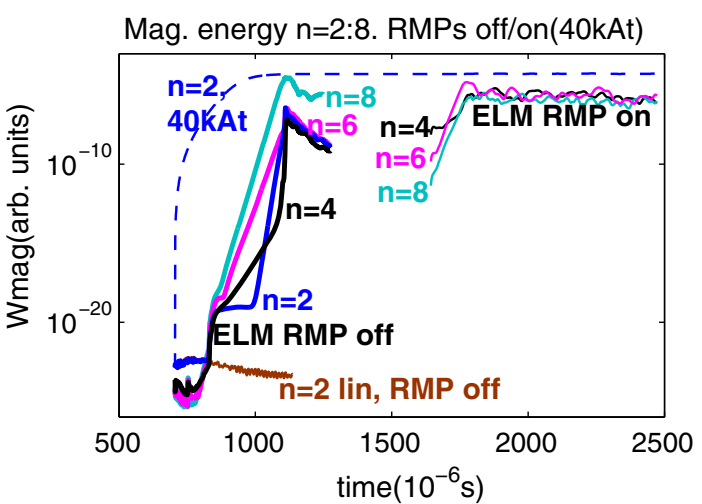

FIG. 1 (color online). Magnetic energy $\left(\sim\left|\psi_{n}\right|^{2}\right)$ of modes $n=$ $2: 8$ in natural and mitigated ELMs with $n=2$ RMP at $40 \mathrm{kAt}$.

Resistivity and viscosity are temperature dependent, the central value of the Lundquist number was taken $S \sim 10^{7}$, which for numerical reasons is lower compared to the experimental value $\left(S=10^{9}\right)$. In the typical JOREK run, initially the equilibrium with flows is obtained for the toroidal harmonic $n=0$ (axisymmetric component) on a time scale of $\sim 1 \mathrm{~ms}$ [18]. After that, for ELMs modeling, other harmonics are initialized in plasma at "noise" level $\left(\sim 10^{-25}\right)$. In the case of RMPs vacuum amplitude for corresponding harmonic (here $n=2$ from error-field correction coils (EFCC) $[6,17,19]$ ) is set at the boundary and increased in time until the stationary conditions are reached [18], resulting in a three-dimensional equilibrium, then other harmonics are initialized to study interaction of ELMs with RMPs.

Main results. - In Fig. 1 the time evolution of the magnetic energy is presented for two cases. The first one corresponds to the nonlinear modeling of an unmitigated ELM where the harmonics $n=0$ and $n=2,4,6,8$ were taken into account. As appeared after numerical tests (not presented) $n>8$ modes growth rates were smaller due to the diamagnetic stabilization [14], so they were excluded from modeling to save computational time. The axisymmetric component $n=0$ is coupled nonlinearly to all harmonics permitting self-consistent modeling of the profile evolution due to ELMs and RMPs. Note that on the linear stage the most unstable mode is $n=8$; the $n=2$ mode is stable. Approaching the ELM crash (maximum of magnetic energy for $n=8$ ) all modes grow due to the nonlinear coupling [11,21] but $n=8$ remains the largest one. After the crash, the magnetic energy starts to decrease since the transport generated during the ELM [10,11] leads to a relaxation of the edge pressure profile, removing the drive for the ELM instability. Note also that for a linear run (without mode coupling) the single mode $n=2$ remains stable (Fig. 1). In the second case, $n=2$ RMPs are applied at the computational boundary and progressively increased until the magnetic energy of RMP reaches a stationary value. After that, the other modes $n=4,6,8$ are included

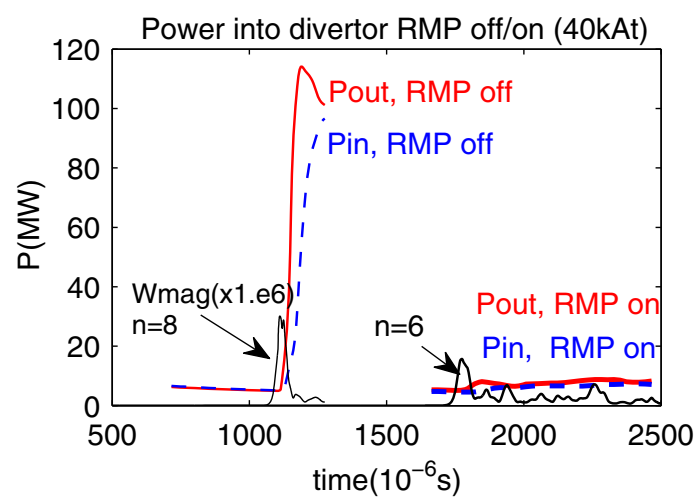

FIG. 2 (color online). Power to inner and outer divertor in natural and mitigated by RMPs $(n=2,40 \mathrm{kAt})$ ELMs.

into the simulation. In this case the behavior of all modes is quite different: the magnetic energies of the $n=4: 8$ oscillate in time with similar maximal amplitudes, much smaller than $n=8$ in unmitigated ELM. The power and heat fluxes on the divertor plates are reduced by about a factor of 10 with RMPs (Fig. 2), corresponding to experiment [5]. The magnetic energy of the modes in the mitigated ELMs decreases while that of the EFCC current increases as illustrated on Fig. 3. The test run with $n=$ $1,2, \ldots 8$ with $\Delta n=1$ without and with RMP confirmed that mitigated ELMs consist of even modes coupled with $n=2$ RMP and the odd modes $(n=1,3,5,7)$ remain on the noise level in spite of the fact that linear growth rates of $n=8$ and $n=7$ modes were very similar in ELM without RMPs (not presented). The initial energies for even harmonics increase with the EFCC current, due to the stronger coupling with $n=2$ RMP (Fig. 4), because they are dominant with RMPs compared to odd harmonics, but the question is why ballooning modes $(n=7,5)$, which were unstable without RMPs, are not developing? The first hypothesis we tested was that the enhancement of the edge transport by RMPs reduces pressure gradient and hence growth rates of the modes and consequently the ELM size. Yet, a simulation without RMPs at artificially

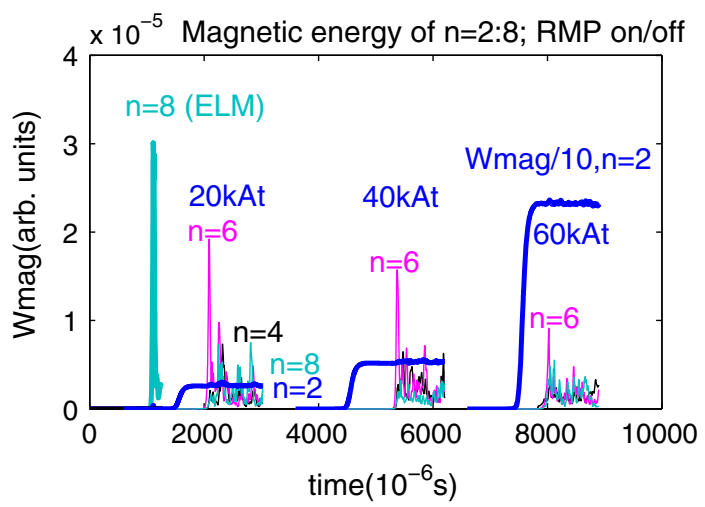

FIG. 3 (color online). Magnetic energy of modes $n=2: 8$ in natural and mitigated ELMs at EFCC current scan. 


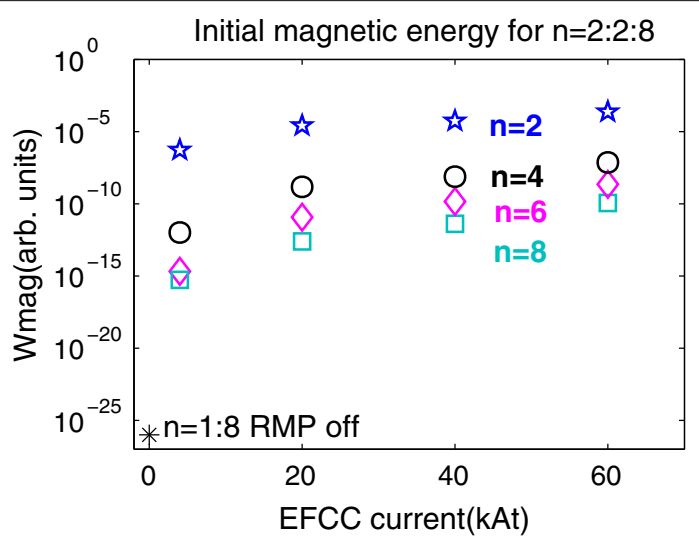

FIG. 4 (color online). Initial magnetic energy of even harmonics coupled to $n=2$ RMP.

lowered pressure gradient (down to a level corresponding to the pressure gradient with RMPs- "low P" on Figs. 5-6demonstrates only a slight reduction of the $n=4: 8$ mode growth rates in the linear stage (Fig. 6), but the $n=8$ mode remains the most unstable and produces a large ELM (not presented here). The detailed analysis of the time evolution of the growth rates of the mode $n=8$ in the early (linear) stage is presented in Fig. 6 for different cases. The time traces for each case were shifted in time correspondingly in order to compare the growth rates before the first relaxation in each run. During the exponential growth of the mode, the growth rate is almost constant which is the case for the unmitigated ELM, at lower pressure gradient (low $P$ ) without RMPs and at low RMP amplitude (4 kAt). At larger RMPs (> $20 \mathrm{kAt})$, the strictly speaking "linear" stage of the $n=8$ mode does not exist anymore. The growth rates oscillate in time, leading on average to an even slower growth of the mode. In the case when threedimensional magnetic topology with RMP at $40 \mathrm{kAt}$ is established and then $n=4-8$ modes are initialized, the $n=8$ mode grows similarly as in an unmitigated ELM if the nonlinear coupling for $n=8$ mode is switched off in the code (case "40 kAt, lin" on Fig. 6). Summarizing, this

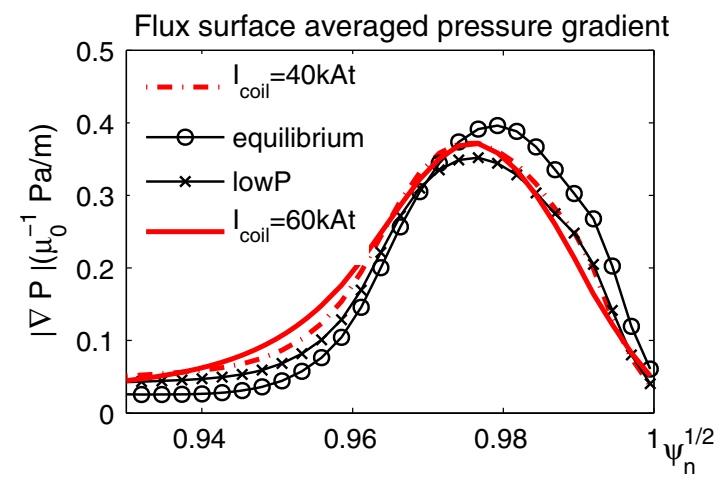

FIG. 5 (color online). Flux averaged edge pressure gradient before an ELM without RMP, at lower initial pressure without RMP, and at 40 and $60 \mathrm{kAt}$ EFCC current.

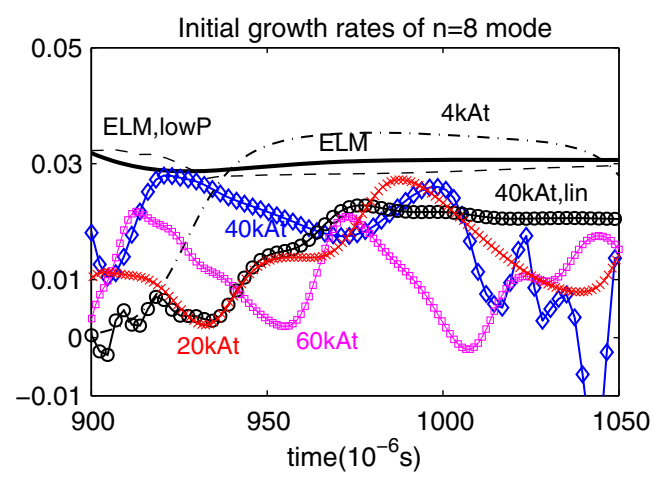

FIG. 6 (color online). The initial growth rate of $n=8$ mode in natural ELM (bold), at lower pressure gradient (dashed), with RMP at 4 kAt (dot-dashed), at $20 \mathrm{kAt}$ (cross), at $40 \mathrm{kAt}$ (diamonds), at $40 \mathrm{kAt}$ in linear run for $n=8$ (three-dimensional equilibrium) in circles, at $60 \mathrm{kAt}$ (squares).

analysis suggests that neither the reduced pressure gradient nor the modified three-dimensional magnetic topology induced by RMPs leads to ELM mitigation without nonlinear coupling: each only slightly reduce the linear growth rate of the mode $n=8$, but the large ELM due to $n=8$ mode still happens with a certain time delay. Only the case with the nonlinear coupling of the modes demonstrated the ELM mitigation. The emerging physical picture we propose here is the following: in natural ELM, the most unstable mode (here $n=8$ ) should reach a sufficiently large level of perturbations to create conductive heat transport along perturbed magnetic field lines and convective $E \times B$ density transport for the profile relaxation in ELM [10,11], where $E$ and $B$ are electric and magnetic fields, respectively. If externally applied RMP (here $n=2$ ) amplitude is not strong enough in plasma, the transport due to RMPs only reduces the growth rates of the modes, but it is not sufficient for the pedestal to become completely stable for ballooning or peeling modes, and the mitigated ELM regime occurs. The modes most strongly coupled to RMP are growing from an already initially large amplitude

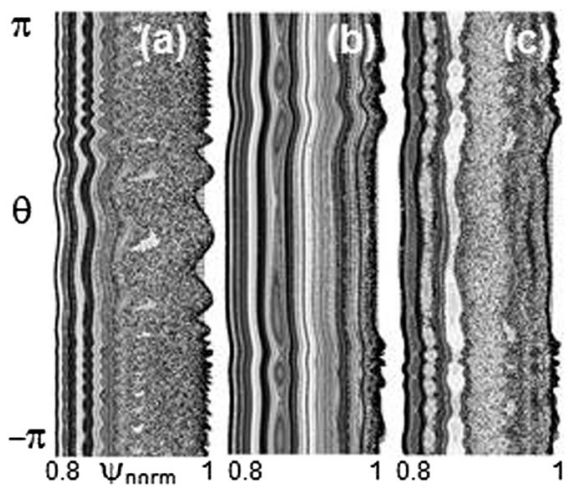

FIG. 7. Edge magnetic topology (Poincaré plot) for natural ELM due to $n=8$ mode (a), with RMP $n=2,40$ kAt (b), with mitigated by RMPs ELMs (c). Coordinates are geometrical poloidal angle and normalized poloidal flux. 


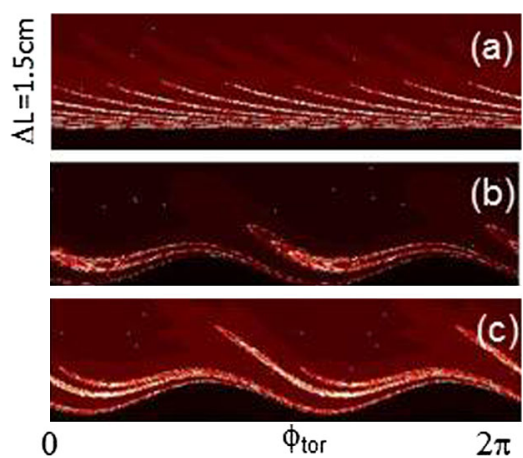

FIG. 8 (color online). Footprints in the outer divertor in the natural $n=8$ ELM (a), with RMP $n=2$ (40 kAt) (b), with mitigated ELMs (c). Brighter colors indicate longer connection length.

and quickly reach a sufficient level to produce additional transport to the SOL compared to the transport produced by RMPs only. Hence, RMP is a constant strong nonlinear drive for these modes. The magnetic energy now is distributed between the modes $n=2,4-8$, increasing energy in $n=2,4,6$ harmonics and decreasing energy of the $n=8$ mode compared to the natural ELM case. Thus the ELM energy "cascades" nonlinearly toward lower $n$ numbers [22], observed in KSTAR RMP experiments [23]. As a result the relaxations due to the multimodes can manifest themselves as more frequent small ELMs or MHD turbulence similar to type II ELMs, providing sufficient transport and hence preventing large ELM crash due to a single most unstable mode that has no time to grow. It is important to note that with RMPs the ballooning nature of the modes changes to tearinglike parity $\left(\psi_{n, m} \neq 0\right.$ on the corresponding rational $q=m / n$ surface as compared to the ballooninglike parity $\left(\psi_{n, m}=0\right.$ on $\left.q=m / n\right)$ [22]), since they are driven by RMPs. It is clearly seen from the comparison of the edge magnetic topology (Fig. 7). For the case of the natural ELM one observes typical ballooning distortion of the magnetic surfaces due to mode $n=8$ [Fig. 7(a)]. The corresponding "footprints" in the outer divertor show a clear $n=8$ structure [Fig. 8(a)]. The magnetic topology with $n=2$ RMPs [Fig. 7(b)] indicates that only the very edge is ergodic: this is due to the rather strong screening of RMP rotation in the pedestal region [18]. The corresponding footprints [Fig. 8(b)] show a typical static $n=2$ structure. In the ELM regime mitigated by RMP with $n=2$, the very edge keeps mainly an $n=2$ structure, but a new island $(m / n=9 / 4,14 / 6$, and 15/6) occurs on the corresponding rational surfaces [Fig. 7(c)]. The corresponding footprints keep the $n=2$ structure imposed by external RMPs, being modulated by the presence of $n=4,6,8$ modes [Fig. 8(c)]. Note that similar footprints of ELMs with RMPs were reported in experiments [24]. This Letter is essentially devoted to the description of the mitigated by RMPs ELM regimes. Note however that the total ELM suppression can be

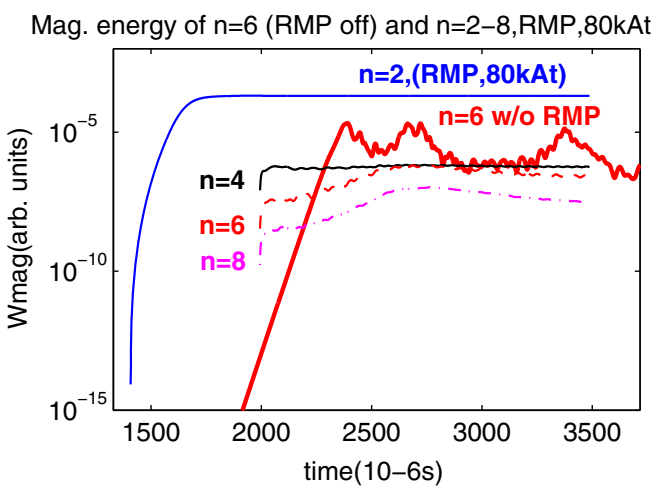

FIG. 9 (color online). Magnetic energy in ELMs due to $n=6$ mode without RMP (in bold) and magnetic energy in $n=2: 8$ modes in total ELM suppression regime with RMP $n=2$ at 80 kAt.

obtained if RMP amplitude in plasma is large enough to produce sufficient transport reducing the pedestal pressure gradient to the values when peeling-ballooning modes are totally stabilized leading to the total ELM suppression regime [3]. The case of total ELM suppression is presented in Fig. 9 for slightly different plasma parameters, where the diamagnetic term was artificially increased by $\sim 10 \%$ and natural ELM was due to the most unstable mode $n=6$. Characteristic ELM cycles due to $n=6$ mode relaxation observed without RMPs disappear with RMP $n=2$ at 80 kAt (Fig. 9).

Conclusions.-ELM mitigation by RMPs was demonstrated in the nonlinear resistive MHD modeling using the JOREK code $[10,18]$. Realistic JET plasma parameters and the RMP spectrum of EFCC $(n=2)$ were used. In unmitigated ELM, medium $n=8$ was the most unstable mode, producing a large ELM crash. The mitigated ELMs consist of the modes nonlinearly driven by RMPs, which have a tearinglike structure, generating islands and additional to RMP edge ergodization, leading to the continued transport from the pedestal to SOL. Mitigated ELMs represent more frequent small relaxations compared to a large "natural" ELM crash. The divertor power flux is reduced by almost a factor of 10 by RMPs. Divertor footprints of mitigated ELMs exhibit mainly structures created by $n=2$ RMPs, modulated by other low $n$ modes. The mechanism of ELM mitigation by RMPs proposed and modeled here reproduces many generic features of high collisionality ELM mitigation regimes observed in experiment, where large ELMs are replaced by small frequent ELMs or broadband magnetic turbulence [3-5,9,24]. Total ELM suppression was also demonstrated in modeling at higher RMP amplitude.

This work has benefited from financial support from the National French Research Program (ANR): ANEMOS (2011) and E2T2(2010) and from the Grant Agency of the Czech Republic under Grant No. P205/11/2341. The views and opinions expressed herein do not necessarily 
reflect those of the ITER organization. This work was granted access to the HPC resources of Aix-Marseille University financed by the project Equip@Meso (ANR10-EQPX-29-01). A part of this work was carried out using the CCRT-CURIE supercomputer within project GENCI (gen2197) and PRACE (ra1904) and the HELIOS supercomputer system (IFERC-CSC), Aomori, Japan, under the Broader Approach collaboration, implemented by Fusion for Energy and JAEA.

[1] R. Hawryluk et al., Nucl. Fusion 49, 065012 (2009).

[2] A. Loarte et al., Plasma Phys. Controlled Fusion 45, 1549 (2003).

[3] T. E. Evans et al. Phys. Rev. Lett. 92, 235003 (2004).

[4] W. Suttrop et al., Phys. Rev. Lett. 106, 225004 (2011).

[5] Y. M. Jeon et al., Phys. Rev. Lett. 109, 035004 (2012).

[6] Y. Liang et al., Phys. Rev. Lett. 98, 265004 (2007).

[7] J. M. Canik et al., Nucl. Fusion 50, 034012 (2010).

[8] A. Kirk, J. Harrison, Yueqiang Liu, E. Nardon, I. T. Chapman, and P. Denner, Phys. Rev. Lett. 108, 255003 (2012).

[9] M. Fenstermacher et al., Phys. Plasmas 15, 056122 (2008).

[10] G. T. A. Huysmans, S. Pamela, E. van der Plas, and P. Ramet, Plasma Phys. Controlled Fusion 51, 124012 (2009).

[11] G. T. A. Huijsmans and A. Loarte, Nucl. Fusion 53, 123023 (2013).
[12] S. Pamela, G. T. A Huijsmans, A. Kirk, I. T. Chapman, J. R. Harrison, R. Scannell, A. J. Thornton, M. Becoulet, and F Orain, Plasma Phys. Controlled Fusion 55, 095001 (2013).

[13] L. E. Sugiyama and H. R. Strauss, Phys. Plasmas 17, 062505 (2010).

[14] P. W. Xi, X. Q. Xu, and P. H. Diamond, Phys. Rev. Lett. 112, 085001 (2014).

[15] H. Strauss, L. Sugiyama, G. Y. Park, C. S. Chang, S. Ku, and I. Joseph, Nucl. Fusion 49, 055025 (2009).

[16] N. Ferraro, T. E. Evans, L. L. Lao, R. A. Moyer, R. Nazikian, D. M. Orlov, M. W. Shafer, E. A. Unterberg, M. R. Wade, and A. Wingen, Nucl. Fusion 53, 073042 (2013).

[17] M. Becoulet et al., Nucl. Fusion 52, 054003 (2012).

[18] F. Orain et al., Phys. Plasmas 20, 102510 (2013).

[19] M. Becoulet, Nucl. Fusion 48, 024003 (2008).

[20] F. L. Waelbroeck, I. Joseph, E. Nardon, M. Bécoulet, and R. Fitzpatrick, Nucl. Fusion 52, 074004 (2012).

[21] I. Krebs, M. Hölzl, K. Lackner, and S. Günter, Phys. Plasmas 20, 082506 (2013).

[22] D. Biskamp, in Nonlinear Hydrodynamics, Cambridge Monographs on Plasma Physics Vol. 1 (Cambridge University Press, New York, 1993), Chap. 7.

[23] G. S. Yun W. Lee, M. J. Choi, J. Lee, H. K. Park, B. Tobias, C. W. Domier, N. C. Luhmann, A. J. H. Donné, and J. H. Lee, Phys. Rev. Lett. 107, 045004 (2011).

[24] M. W. Jakubowski, Nucl. Fusion 49, 095013 (2009). 